\title{
Beauté, ordre et désordre vestimentaires féminins en Grèce ancienne
}

Beauty, Order and Disorder in Women's Clothing in Ancient Greece

\section{Florence Gherchanoc}

\section{(2) OpenEdition \\ 1 Journals}

Édition électronique

URL : http://journals.openedition.org/clio/10717

DOI : $10.4000 /$ clio. 10717

ISSN : 1777-5299

Éditeur

Belin

Édition imprimée

Date de publication : 31 décembre 2012

Pagination : 19-42

ISSN : 1252-7017

Référence électronique

Florence Gherchanoc, «Beauté, ordre et désordre vestimentaires féminins en Grèce ancienne », Clio.

Femmes, Genre, Histoire [En ligne], 36 | 2012, mis en ligne le 31 décembre 2014, consulté le 30 avril 2019. URL : http://journals.openedition.org/clio/10717 ; DOI : 10.4000/clio.10717

Tous droits réservés 


\title{
Beauté, ordre et désordre vestimentaires féminins en Grèce ancienne
}

\author{
Florence GHERCHANOC
}

«Car, pour ce qui concerne l'habillement, le peuple ne se distingue pas des esclaves et des métèques et son apparence extérieure n'est pas meilleure $»^{1}$. C'est en ces termes que l'auteur d'un pamphlet contre la démocratie se plaint du dérèglement vestimentaire, une des manifestations du désordre politique qui règne dans la cité athénienne à la fin du Ve siècle avant notre ère. Il suggère que la situation est plus satisfaisante à Sparte, présentée comme un contre-modèle, puisque l'on y différencie au premier coup d'œil, grâce à leur costume, les citoyens de leurs dépendants ruraux, les hilotes. Les premiers portent des habits tissés, les seconds des vêtements confectionnés avec des peaux de bêtes, moutons et chèvres - dont la fameuse katônakê, un vêtement grossier, servile et paysan -, signe qu'ils n'appartiennent pas au monde civilisé 2 .

Ce constat relatif à l'indifférenciation et à la neutralité vestimentaires supposées des hommes de l'Athènes classique pourrait-il également s'appliquer plus largement à la gent féminine? Pour esquisser une réponse à cette question, il convient de réfléchir à « ce que pourrait être à un moment donné un système vestimentaire et l'ensemble axiologique qui le constitue (contraintes, interdictions, tolérances, aberrations, fantaisies, congruences, exclusions) $»^{3}$. Marqueur visible

* J'adresse mes remerciements les plus chaleureux à Catherine Baroin, qui a relu attentivement ce texte, pour ses remarques et suggestions avisées.

[Xénophon], Constitution des Athéniens, I, 10.

2 Myron de Priène apud Athénée, XIV, 657D (FGrH 106 F 2). Cf. Ducat 1974 : 1455-1457.

3 Barthes $1957: 432$. 
d'identité, le vêtement révèle autant qu'il cache. Il est le signe et la garantie des corps sexués, du statut politique, social, économique et religieux des personnes. Ainsi, les gestes et les attitudes corporelles liés aux façons de se vêtir ou de se dévêtir relèvent aussi bien de comportements attendus et reconnus par un groupe, par une société donnée, que de stratégies de distinction particulières ${ }^{4}$. Dans cette perspective, je m'intéresserai au(x) costume(s) féminin(s) comme langage, à l'apparence ou "présentation de soi» dans leurs dimensions sociologique et culturelle dans le monde grec ancien. Précisément, je me demanderai si le vêtement fait la femme et surtout quelle femme (la fille, l'épouse, l'hétaïre, la vertueuse, la dévergondée, etc.). L'enveloppe vestimentaire, doublet ou encore prolongement du corps, est-elle fonction de son âge, de la conjoncture (domestique, politique, rituelle, etc.), de sa place et de son rang dans la société ? Quels sont les liens entre vêtement et beauté ? L'ensemble conduira à une réflexion sur l'existence d'un costume «grec» typiquement féminin et sur son statut.

À partir d'exemples significatifs, je m'attacherai ainsi à l'analyse de discours produits à propos de vêtements reconnus comme féminins et montrerai comment en fonction des contextes et des activités, le costume qualifie les femmes et opère finalement des hiérarchies.

\section{À la recherche d'un « éternel [costume] féminin "}

Quoi de mieux pour « reconstruire» un costume féminin archétypal que d'observer ce que revêtent les hommes quand ils veulent se faire femmes? Deux scènes de travestissement de l'époque classique à Athènes nous renseignent sur ce point.

La première a pour cadre les Thesmophories d'Aristophane, une comédie datée de 411 avant notre ère. Alors que les femmes d'Athènes célèbrent la fête en l'honneur de Déméter où les épouses de citoyens agissent pour la prospérité de la cité, Euripide, pour plaider sa cause auprès de ces dernières, cherche un homme à déguiser en femme qui se glisserait parmi elles. Il pense d'abord au

4 Sur les symboliques du vêtement en histoire ancienne, voir notamment Losfeld 1991 ; Llewellyn-Jones (ed.) 2002 ; Cleland, Harlow \& Lewellyn-Jones (eds) 2005 ; Gherchanoc \& Huet 2007. 
poète tragique Agathon en raison de son allure efféminée, avant que le rustre Mnésilochos, un parent par alliance, ne se propose pour cette mission. Ce dernier, une fois « flambé et épilé », est habillé d'une robe safran (krokôtos), d'un soutien-gorge (strophion), d'un manteau arrondi (egkuklon), d'un tour de tête (kephalê perithetos) à la place de la résille (kekruphalos) et du bandeau (mitra), et de chaussures rigides. Ainsi dissimulé par des vêtements, il a l'apparence (eidos) - autrement dit, le costume - d'une femme. Il lui reste à se comporter et à parler comme une femme (gunaikizein) $)^{5}$.

La seconde est tirée des Bacchantes d'Euripide, une tragédie composée en 408 et représentée en 405 à Athènes. Elle raconte comment Penthée, roi de Thèbes, déguisé par Dionysos qu'il ne reconnaît pas comme dieu, a porté « une tenue de femme, de ménade, de bacchante (skeuê gunaikos mainados bachês) » ${ }^{6}$ et épié les femmes, avant d'être pris par ces dernières et de finir démembré. Pour mieux les espionner, il revêt une robe de lin (bussinoi peploi), un vêtement aux longs plis (peploi podêreis), place sur sa tête une «longue perruque », sur le "front une mitre ", puis il ajoute à cet attirail, pour se façonner un parfait costume rituel et se faire ménade, le thyrse et la nébride, une peau de faon tachetée, des emblèmes dionysiaques. Ce faisant, il ressemble à sa mère et à ses tantes?

Ainsi, les éléments du costume féminin se déclinent de la tête aux pieds : des bandeaux, des filets et tours de tête, et, surtout, pour couvrir le corps, la crocote, le strophion et les peploi, caractéristiques de la parure féminine (au moins à Athènes), enfin des chaussures. Le vêtement dissimule le corps tout en construisant et définissant une identité de genre. Plus que les traits du visage (un teint clair, l'absence de poils et des cheveux longs) ou la physionomie générale et la voix, des habits spécifiques sont clairement des marqueurs normés de l'identité sexuée, y compris dans le cas de la ménade. Le costume, signe et garantie de la distinction des corps sexués, établit bien des distinctions de genre.

\footnotetext{
Aristophane, Les Thesmophories, 214-268.

Euripide, Les Bacchantes, 915.

Ibid., 821 sq. Sur le travestissement de Penthée, voir Gherchanoc 2003 : 745-748.
} 
Dans le monde grec ancien, on oppose ainsi le corps féminin empaqueté, empêtré et caché dans de longs vêtements au corps masculin, paré de sa plus simple nudité - un costume de tissus musculaires - ou d'une nudité partielle en fonction des lieux et des situations (au gymnase, à la guerre, etc.) ${ }^{8}$. Pourtant le vêtement féminin est relativement simple. Il est constitué d'une tunique, des peploi, généralement en laine, ou d'un chiton (sans manches, à manches courtes ou à manches longues), en lin ou bien en laine. Ces pièces de tissu rectangulaire, qui tombent jusqu'aux pieds, sont fermées aux épaules ou attachées aux bras par deux fibules, des boutons ou des points de couture de part et d'autres du cou, et ceinturées à la taille. Dessus, les femmes revêtent éventuellement un grand manteau rectangulaire de laine, le plus souvent un bimation, drapé autour du corps. La tête et les épaules peuvent également recevoir un voile (kaluptron $)^{9}$. Les pieds sont chaussés de sandales ou de chaussons, à talons plus ou moins hauts.

Le costume féminin se distingue en outre par son agencement et par la qualité des étoffes, par leur nature, leur couleur et leur ornementation, autrement dit par leur richesse. Il diffère enfin suivant le statut, l'âge et le contexte. Aussi les vêtements les plus communs, pour la vie de tous les jours, ne sont-ils probablement pas les mêmes que les parures de séduction ou encore les tenues rituelles portées lors des fêtes.

\section{Quand Zeus créa la femme : un costume de séduction}

Pour les Grecs, depuis Hésiode (VIII siècle avant notre ère), la femme est dénuée de corps et se définit par ses atours. La parure est, dans la Théogonie, ce qui constitue la femme comme un beau dehors ${ }^{10}$, à l'image des déesses, en particulier Aphrodite, dont elle a l'apparence

8 Cf. Aristophane, Lysistrata, 661-663. Voir Iriarte 2006 : 289-301 ; Gherchanoc $2008: 75-101$.

9 Barker 1922; Morizot 2001; Llewellyn-Jones (ed.) 2002; Jeammet 2003; Llewellyn-Jones 2003 ; Bectarte 2009 : 236-238.

10 Hésiode, Théogonie, 571-589. Voir Loraux 1989 : 150. 
$(e i d o s)^{11}$. Façonnée sur ordre de Zeus, Pandora est modelée par Héphaïstos, puis habillée par Athéna. La fille du Cronide

lui noua sa ceinture (zôse), après l'avoir parée d'une robe blanche (kosmêse [...] argupheêi esthêtz), tandis que de son front ses mains faisaient tomber un voile aux mille broderies (kaluptrên daidaleên) [...]. Autour de sa tête, elle posa un diadème d'or (stephanên chruseên) forgé par l'illustre Boiteux lui-même $[\ldots]$ : il portait d'innombrables ciselures $[\ldots]$ - et un charme infini illuminait le bijou (charis d'ampelampeto pollê) $[\ldots]^{12}$.

Le costume de Pandora comprend un vêtement étincelant de blancheur et ceinturé, un beau voile brodé et un bijou d'or pour la tête. Superbement parée (kosmô agallomenên), elle est ainsi «merveille pour les yeux ». Les attributs de l'ancêtre du genos gunaikos (de la race des femmes) forment sans conteste un costume de séduction que synthétise le terme kosmos, à la fois parure et ordre ${ }^{13}$. Il est en même temps, dans sa conception, celui d'une jeune fille nubile ou d'une numphê, une épousée, au jour de ses noces, brillante de charis (grâce), resplendissante de beauté, qui doit émerveiller: une fille née/créée pour le mariage ${ }^{14}$. Les numphai sont, en effet, parées de la plus belle façon pour leurs noces et exposées aux yeux de tous comme «objets érotiques », notamment lors du repas et de la procession nuptiale. Elles doivent éblouir le fiancé et l'assistance, susciter un puissant désir, permettre l'attraction sexuelle et la mixis (réunion) des corps ${ }^{15}$. À l'acmé de leur vie, étincelantes, elles sentent bon et sont belles à voir, ornées d'étoffes aux teintes somptueuses et variées ${ }^{16}$. Ce costume dit et renforce leur sex appeal. Tissus diaprés (peploi poikilor), parfums et bijoux d'or ciselés constituent une beauté sociale, qui est canalisée pour la

11 Pirenne-Delforge 2001 : 98-99.

12 Hésiode, Théogonie, 571-584. Cf. Hésiode, Les travaux et les jours, 70-76.

13 Sur le terme kosmos, voir Casevitz 1989 et Cartledge, Millet \& von Reden (eds) 1998.

14 Des parures du même type caractérisent d'autres figures féminines dans des contextes qui mêlent beauté, charme, sexualité et érotisme. Celles d'Héra et d'Aphrodite en constituent les archétypes divins. Cf. Homère, Iliade, XIV, 170189; 214-217 ; Hymne bomérique à Aphrodite I, 61-66 ; 84-90; 162-164 ; Hymne homérique à Aphrodite II, 5-18.

15 Voir Gherchanoc 2012 : 134-136.

16 Cf. Aristophane, Ploutos, 529-530. 
communauté en vue de la réussite du mariage. Le costume annonce ce qu'attend la cité de ces jeunes filles. Ainsi transformées en thaumata (objets d'admiration) pour les sens, elles incarnent de dignes épouses en devenir, séductrices pour un époux, mais pleines de pudeur et de tempérance, et des mères en puissance, productrices d'enfants légitimes pour le bien de l'oikos (famille) et de la cité.

Le costume des jeunes filles est ainsi à l'interface d'une séduction façonnée et factice, d'une beauté «ajoutée $»^{17}$, et d'une beauté propre à leur âge, à leur statut et à leur état, qui sont mobilisées au service de la cité. Le corps paré et animé de Pandora en constitue le "prototype » : il suscite une "stupeur admirative» et un "élan de désir amoureux $»^{18}$. Pour celles dont le destin est d'être données en mariage, il définit qui elles sont et leur place dans les sociétés grecques.

Ce costume de séduction reste aussi, plus tard, celui des épouses accomplies qui ont encore à plaire, mais à leurs seuls époux et dans un contexte exclusivement privé cette fois. Aristophane en joue dans ses comédies et les dépeint maquillées, des «tuniques safranées sur le dos, bien attifées avec des cimbériques tombant droit [tuniques droites non cousues, ouvertes et sans ceinture] et des péribarides [chaussures légères d'intérieur]» ou encore vêtues de leurs "petites tuniques d'Amorgos ", " nues, le delta épilé ». Cette tenue féminine dit leur disponibilité sexuelle. Elle renvoie à la séduction et à l'érotisme, et devient même l'arme d'une guerre domestique dans Lysistrata ${ }^{19}$.

\section{Un costume rituel}

La bigarrure, les couleurs tranchées et la brillance des vêtements rituels des numphai sont aussi caractéristiques d'autres parures rituelles féminines, des jeunes et des adultes. Le costume des épousées est, à cet égard, assez comparable à celui des canéphores, ces jeunes filles en âge d'être mariées, que l'on exhibe dans les célébrations de la cité. Belles, les joues blanchies, elles portent un vêtement bariolée ${ }^{2}$. Comme le rappelle le demi-chœur des femmes athéniennes dans

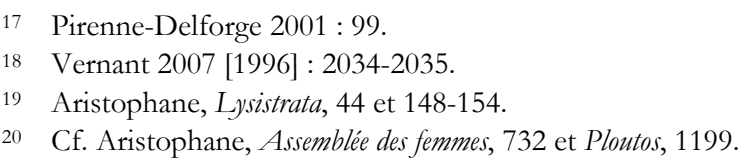


Lysistrata, ce costume comprend des "[t]apisseries chatoyantes (strômata poikila), [des] mantelets de laine (chlanidia), [des] tuniques fines (xustides), [des] bijoux en or (chrusia) $»^{21}$. Le spectacle de la beauté radieuse des jeunes filles ainsi vêtues signale à quel point les canéphores constituent un "moyen d'échange et d'alliance » pour les pères qui les offrent aux regards des prétendants et une promesse de prospérité pour la cité qui, par leur intermédiaire, rend hommage aux dieux ${ }^{22}$. À Sparte, au même âge, les filles offrent, en revanche, aux yeux de tous, en guise de spectacle, le costume de leur nudité ou semi-nudité, pour les mêmes raisons ${ }^{23}$.

Une tablette en bois, trouvée à Pitsa en Achaïe et datant de 530520 avant notre ère, nous donne une idée de ce qu'a pu être ce costume coloré (Cf. Cabier des illustrations, figure 1). Des jeunes femmes sont figurées dans une procession. Elles sont vêtues d'une longue tunique bleue agrémentée de bordures en quatre endroits (au niveau du cou, de l'estomac, à la taille, et à hauteur des pieds), recouverte par un manteau rouge, à l'exception de la dernière qui semble totalement enveloppée dans un vêtement bleu. La première porte le kanoun (panier) et verse une libation, sa chevelure relevée est retenue par une couronne en feuillage d'or. Les deux autres, à peine plus âgées, ont les cheveux frisés et lâchés maintenus par un bandeau ceint sur la tête et une couronne comparable à celle de la canéphore. Elles sont belles et leur vêtement renforce leur éclat et celui de la célébration en l'honneur des nymphes.

En effet, en vue de célébrer les dieux de la cité, dans de nombreux cultes, le port de costumes très raffinés est autorisé, voire préconisé. Ainsi, à l'époque archaïque, les femmes de Corinthe honorent Héra vêtues de leurs plus beaux vêtements, comme en témoignent deux épisodes bien connus de la vie de Périandre (fin du VII siècle avant notre ère) racontés par Hérodote, où le tyran les agresse après la mort de son épouse. Dans une version de l'histoire, suivant

[...] le conseil de Thrasybule [qui] était de mettre à mort les citoyens qui dépassaient les autres, [...] en une seule journée il fit dépouiller (apeduse)

21 Aristophane, Lysistrata, 1189-1193. Voir Brulé 1987 : 301-302 ; Roccos 1995.

Bruit Zaidman 1996 : 38, 41

23 Plutarque, Lycurgue XIV, 3-4 et 7 ; XV, 1. Voir Gherchanoc 2008 : 93-96. 
[de leurs vêtements] toutes les femmes des Corinthiens en l'honneur de sa propre femme Mélissa [...].

Dans l'autre, pour révéler l'emplacement d'un trésor, le spectre de Mélissa demande à être enterrée avec des vêtements brulés suivant l'usage.

[A]ussitôt après le message reçu, [Périandre] fit ordonner par une proclamation que toutes les femmes des Corinthiens se rendissent hors de la ville au temple d'Héra. Elles y allèrent, comme pour une fête, parées de leurs plus beaux atours (kosmô tô kallistô chreômenai) : mais lui, qui avait aposté ses gardes, les fit dépouiller pareillement, femmes libres et servantes, fit porter les dépouilles en monceau dans une fosse et les y fit brûler pendant qu'il priait Mélissa ${ }^{24}$.

Ces très beaux ornements féminins, dont sont dépossédées les femmes, sont probablement composés de vêtements fins, éclatants, lumineux et blancs, ou colorés, de pourpre par exemple, ainsi que de bijoux d'or ${ }^{25}$. La description faite par l'historien Douris (340-270 avant notre ère), citant le poète Asios (VII siècle avant notre ère), d'une procession en l'honneur d'Héra à Samos, au sujet du luxe excessif (truphê) des hommes comme des femmes, le suggère aussi :

Ils portaient des bracelets (chlidônes) autour de leurs bras. Et quand ils célébraient Héra, ils marchaient avec leurs longs cheveux soigneusement tressés leur tombant sur la poitrine et les épaules [...] enveloppés dans leurs beaux vêtements (pepuskasmenoi heimasi kalois), leurs tuniques neigeuses (chioneoi chitônes) effleurant le sol de la vaste terre, une barrette en or accrochée, telle une cigale, à leurs cheveux et leurs rubans dorés (chruseiai korumbai) ondulant au gré de la brise, des bracelets finement ouvragés (daidalees chidônes) cerclant leurs bras ${ }^{26}$.

À Samos, hommes et femmes ornent leurs corps pour célébrer Héra. À Corinthe, pour Périandre, dépouiller les femmes de

24 Hérodote, V, 92G. Cf. aussi, pour une autre tradition, Éphore apud Diogène Laërce, Vie, doctrines et sentences des philosophes illustres, I, 7, 96. Voir Will 1955 : 441571 ; Loraux 2009 [2003].

25 Sur la pourpre et l'or comme symboles de richesse et d'excellence, voir Jones 1999 : 252 et Grand-Clément 2011b : 266-340.

26 Douris de Samos apud Athénée, XII, 525E-F (FGrH 76 F 60). Sur la richesse du costume rituel féminin, cf. Aristophane, Les Thesmophories, 894, où Mnésilochos est accusé de s'être introduit parmi les femmes durant la fête en l'honneur de Déméter afin de voler leur or. 
magnifiques parures de ce type est conforme à la politique de lutte contre le luxe de l'aristocratie, si caractéristique des tyrans archaïques. Ces actes sont également révélateurs d'un mauvais comportement, de l'impiété du tyran et donc d'un dysfonctionnement politique.

Le costume de fête est le plus souvent élaboré, richement orné, en particulier celui des femmes, comme le signale encore un passage de l'Économique de Xénophon (IV siècle avant notre ère). Il y est question, en effet, des habits de l'homme et de la femme qui sont unis dans le mariage en vue de bien gérer l'oikos et de le faire fructifier par leur association ${ }^{27}$. L'époux dispose de deux tenues spécifiques, des vêtements pour les fêtes et pour la guerre, en plus de son costume ordinaire, l'épouse, d'une seule, une parure de fête (kosmos). Le costume de fête féminin est ainsi conçu à la fois comme une mise en ordre et un ornement - kosmos -, tandis que celui de son conjoint est un simple vêtement (esthês). Il est donc une organisation structurée de divers éléments assemblés sur le corps. Cet assemblage crée une beauté ordonnée qui se veut totale et immuable.

La beauté éblouissante et agissante de l'habit de fête féminin contribue à la splendeur des célébrations et en assure la réussite. Cependant certains rituels contraignent, au contraire, les femmes à se dépouiller de leurs ornements. Ainsi, des cultes imposent un dénuement rituel en vue de garantir la piété (eusebeia) et le bon ordre (enkosmia) de la cérémonie. En Arcadie, au Ve siècle avant notre ère, porter un vêtement coloré/bigarré et ornementé (zteraion) est interdit dans le sanctuaire de Déméter Thesmophoros ${ }^{28}$. À Patras, en Achaïe, au IIIe siècle avant notre ère, pour les Démétria, il n'est pas permis de porter de l'or pour une valeur supérieure à une obole, ni un vêtement brodé ou pourpre (mêde lôpion poikilon, mête porphurean), ni de se farder de blanc de céruse (metê psêmuthiousthai) ${ }^{29}$. À la même époque, des éléments comparables - bijoux d'or, "vêtement teint de pourpre, ou fleuri (porphureon beimatismon mêde anthinon), ou noir, avec des chaussures, avec une bague»- sont proscrits à Lycosoura, en

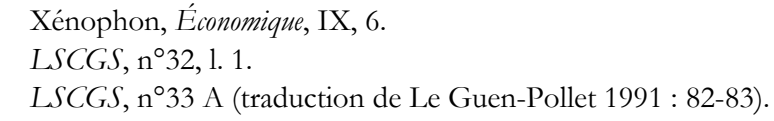


Arcadie, dans le sanctuaire de Despoina ${ }^{30}$. À Andania, en Messénie, vers $91 / 90$ avant notre ère, lors de la célébration, tous ceux qui accomplissent les mystères portent un vêtement blanc (heimatismos leukos) et vont pieds nus. En outre, les costumes féminins sont réglementés de façon encore plus précise pour celles qui participent à la fête et durant la procession :

[Q]ue les femmes ne portent pas de vêtement transparent (mê diaphanê), ni de bordures sur leur manteau plus larges qu'un demi-doigt (mêde ta sameia en tois heimatiois platutera hê) [...]; que les jeunes filles portent un long chiton frangé (kalasêris) ou une fine tunique de lin (sindonitês) et un manteau (beimation) ne valant pas plus d'une mine, et les femmes esclaves un long chiton frangé (kalasêris) ou une fine tunique de lin (sindonitês) et un manteau (beimation) ne valant pas plus de cinquante drachmes. En ce qui concerne les bierai [celles qui ont une charge officielle], que les femmes portent un long chiton frangé (kalasêris) ou une tunique sans bordure (bupoduma mê echon skias) et un manteau (heimation), ne valant pas plus de deux mines; que les jeunes filles portent un long chiton frangé (kalasêris) et un manteau (beimation) ne valant pas plus de cent drachmes. Dans la procession, en ce qui concerne les bierai, que les femmes portent une tunique de dessous (bupodutês) et un manteau épais avec des bordures (beimation ... oulon, sameia) moins larges qu'un demi-doigt, que les jeunes filles portent un long chiton frangé (kalasêris) et un manteau qui ne soit pas transparent (heimation mê diaphanes). Qu'aucune femme ne porte de [parure en] or (mêdemia chrusia), ni de fard rouge ou blanc de céruse (mêde phukos mêde psimithion), ni de bandeau (mêde anadema), ni de cheveux noués (mêde tas trichas anpeplegmenas), ni de sandales (mêde dêmata), si elles ne sont pas en feutre ou en cuir provenant d'animaux offerts en sacrifice aux dieux ${ }^{31}$.

La qualité du costume rituel féminin (sa finesse et ses décorations) est définie; sa valeur en est limitée, en fonction du moment de la cérémonie, du statut des femmes (libre, non-libre), de leur âge (adulte, enfant) et de l'exercice ou non d'une charge officielle ${ }^{32}$. Les éléments qui habituellement contribuent à les embellir de la tête aux pieds et caractérisent leur beauté (tissus fins et transparents ou riches et ornés, fioritures et parures variées) sont fortement réduits. Concernant les contrevenantes, le gynéconome, magistrat chargé des bonnes mœurs dans la cité, est autorisé à arracher leurs belles parures en vue de les

$30 \quad$ IG V 2, 514 ; LSCG, $\mathrm{n}^{\circ} 68$ (traduction de Jost 1985 : 330).

31 IG IV, 1, 1390, 1. 16-26 (traduction modifiée de Deshours 2006 : 30-31).

32 Voir Deshours 2006 : 102-108; Cleland 2009. 
consacrer ${ }^{33}$. Les prescriptions suggèrent, cependant, qu'en d'autres occasions ces mêmes femmes revêtent ce type de costume diaphane et léger, bariolé, brillant et fastueux, portent des bijoux, se maquillent, ont des coiffures sophistiquées, mises en valeur par des accessoires. De plus, ce n'est pas une parure particulière qui est visée. Les éléments fonctionnent collectivement. Le costume féminin dans son ensemble est concerné. Il s'agit dès lors d'imposer aux femmes (épouses et filles de citoyens; esclaves) un costume relativement sobre, des étoffes de valeur limitée et dénuées de luxe excessif, au service et pour le bien de la communautés ${ }^{4}$.

En outre, les Pythagoriciens qui s'opposent aux usages en vigueur dans les cités grecques proposent généralement à leurs fidèles de renoncer au faste vestimentaire pour vivre en adéquation avec la philosophie sectaire. C'est ce que préconise, par exemple, Phyntis, qui vécut au $\mathrm{IV}^{\mathrm{e}}$ ou au $\mathrm{III}^{\mathrm{e}}$ siècle avant notre ère, pour «l'ornement dont il sied d'envelopper le corps » :

Il faut que la femme soit vêtue de blanc, avec simplicité et sans superfluité. Elle y parviendra, si elle n'emploie point, pour se vêtir le corps, des voiles transparents, bigarrés et tissés en soie, mais si elle se sert de tissus modestes de couleur blanche. Ainsi faisant, elle évitera la magnificence, le luxe et la coquetterie, et n'inspirera point une jalousie perverse aux autres femmes. Elle renoncera absolument de porter sur elle de l'or ou de l'émeraude, car ces ornements coûtent très cher et manifestent un orgueilleux dédain pour les femmes du peuple ${ }^{35}$.

Des étoffes fines et chamarrées, des bijoux et autres parures du corps caractérisent ici encore la richesse, l'excellence aristocratique et

33 IG IV , 1, 1390, 1. 26-27. De même, en vertu de la norme, le deuil conduit les femmes à nuire à la beauté de leur corps (lacérations du visage, absence de maquillage, cheveux coupés) et à revêtir de longs peploi foncés. En outre, les règlementations funéraires témoignent de l'attention que portent les cités à limiter et à canaliser, en particulier, l'expression du deuil féminin, par un contrôle du comportement et de la mise. Voir Gherchanoc $2012: 190-203$.

34 Voir Dillon 1997 : 196-199; Dillon 2002 : 261-264 ; Gherchanoc 2011 : 38-39; Grand-Clément 2011a.

35 Fragments et lettres de Theano, de Périctioné, Phintys, Mélissa et Mya (traduction M. Meunier, Paris, L'artisan du livre, 1932) : 71-73. Cf. aussi Diogène Laërce, VIII, 19 et 33 ; Élien, Histoires variées, XII, 32 ; Jamblique, Vie de Pythagore, 100. Pour les Cyniques, cf. Anthologie palatine, VII, 413, à propos d'Hipparchia. 
la beauté fabriquée et factice. Dans tous les cas, quelles que soient les prescriptions religieuses, le costume féminin est instrumentalisé pour garantir l'eusebeia et l'eukosmia, et donc la réussite de la célébration, ainsi que pour préserver l'eunomie (ordre bien réglé) dans la cité. C'est aussi en vue d'éviter la stasis (guerre civile) que les Pythagoriciens promeuvent une certaine forme d'égalité et de discrétion vestimentaires.

\section{Beautés de femmes : de l'eukosmia au désordre vestimentaire}

La mise en ordre du corps féminin par les vêtements et les parures relève d'un art, de la maitrise d'une technê (un savoir-faire technique et savant). Le corps ainsi vêtu et paré est soumis au regard des autres. Il constitue un costume social. Il est une forme de discours et il est luimême objet de discours positifs ou dépréciatifs qui conduisent à jauger, "catégoriser» et hiérarchiser les individus, souvent d'ailleurs par le biais de lieux communs. Les parures deviennent alors parfois un symptôme emblématique de la nature de la femme. Il y a donc coïncidence entre la femme et son costume, qui peut caractériser dans un même mouvement sa beauté et son comportement ou bien signaler et signifier ses défauts.

En effet, la beauté des femmes est équivoque. Canalisée correctement au profit de l'oikos et de la cité, elle est positive. Un bon agencement vestimentaire dit l'ordre et symbolise la beauté du monde. Un accoutrement dépareillé produit l'effet inverse, en disqualifiant des individus mal vêtus au regard des normes culturelles. Ambivalent, le costume féminin est ainsi soit signe de pudeur (aidôs) et de tempérance (sophrosunê), soit la marque d'un comportement rusé, auquel cas il stigmatise. Les discours grecs jouent sur ces deux aspects en fonction de ces attendus.

Une femme pleine de pudeur et tempérée est habillée en conséquence. L'aidôs devient même un vêtement "métaphorique» couvrant son corps: "[e]n même temps qu'elle se dépouille de sa tunique (chiton), une femme se dépouille aussi de son aidôs $»^{36}$. Aidôs et nudité s'opposent. Mais aussi, aidôs et bigarrure. Le vêtement signale

36 Hérodote, I, 8. 
l'excellence comme les défauts féminins. À cet égard, l'apologue de Prodiccos, où deux personnages féminins alimentent la réflexion philosophico-politique de Xénophon sur les qualités d'un bon chef, est instructif. Vices et vertus s'y muent en costumes ${ }^{37}$. Les deux femmes ont la belle et grande stature des déesses et rivalisent pour obtenir les faveurs du héros Héraclès. Ce dernier, ayant atteint l'bêbê, un âge où l'on est maître de son destin, doit déterminer laquelle incarne le mieux la vie qu'il souhaite. Arétè (Mérite) porte un vêtement blanc et non bariolé ; elle n'est pas fardée. Elle ressemble à Pudeur (Aidôs) ou encore à Justice (Némésis) qui, personnifiées, voilent (kalupsamena) leur beau corps (chrôa kalon) d'étoffes blanches (leukoisin pharessi) ${ }^{38}$. Kakia (Mauvaise), elle, est chamarrée et sa beauté, factice. Ses vêtements sont très brillants (esthêta dialampoi) et attirent le regard, son maquillage excessif (d'un blanc et d'un rouge outrés), ses chairs grasses et molles, et sa taille falsifiée. Or le style de vie que chacune d'elles propose à Héraclès est conforme à ses atours, soit une vie de labeur (ponos) et d'application (epimeleia), soit une vie facile et agréable (bê hêdistê te kai rhastê). Chaque costume définit des aptitudes «morales» et comportementales. Celles-ci sont jugées et évaluées suivant un système de valeurs inscrit sur le vêtement, où l'aidôs devient une parure de qualité qui engendre la vraie beauté ; elle est la parure de l'excellence ${ }^{39}$.

Le schéma proposé par Xénophon est d'une certaine façon caricatural et tranché. Parfois, les nuances vestimentaires sont plus ambivalentes. C'est vrai, en particulier, pour le costume des numphai. Ainsi, dans le discours mythique, Pandora est dotée d'une belle apparence (kalon eidos), mais elle est également, en raison de la beauté ajoutée et travaillée de ses atours, ruse trompeuse (dolos), destinée à faire le malheur de la gent masculine ${ }^{40}$. Elle fixe en cela, aux côtés du sacrifice et de l'agriculture, la condition humaine ${ }^{41}$. Elle est, tout

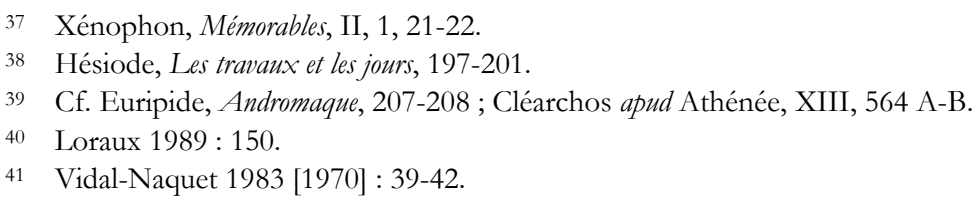


comme son costume un «beau mal» (kalon kakon) ${ }^{42}$, un piège érotique, néfaste pour l'homme et pourtant indispensable à la reproduction de l'espèce humaine par le biais du mariage. C'est pourquoi, durant le mariage, la jeune fille est kalê, belle à voir, parée des plus beaux vêtements pour faire de la fête un spectacle magnifique. Le costume de la mariée lui confère, ce jour, une beauté, bénéfique et agissante (charis), entre pudeur et attrait sexuel. Il fait son pouvoir et assure ainsi la réussite du mariage. La séduction comporte une part de ruse, elle est source de danger comme elle peut se muer en un bienfait: suivant le discours, un même vêtement n'opère pas de la même façon.

En bien des occasions, le costume est le moyen le plus évident pour définir les individus et surtout opposer les catégories. Ainsi, l'épouse légitime a pour négatif vestimentaire la concubine ou encore l'hétaïre ${ }^{43}$. Pour autant, chez elles, les épouses usent de leurs charmes pour séduire leurs maris. Elles revêtent dès lors un costume de séduction qui est le même que celui dont se parent les hétaires dans des contextes comparables, quand elles doivent convaincre de leur beauté et afficher leur disponibilité sexuelle. En outre, peut-on penser que les hétaïres déambulent, en toutes circonstances, dans les rues de la cité, dans un costume très élaboré et riche, dans des tissus transparents et fins ${ }^{44}$ ? Plus vraisemblablement, le costume agit comme un symbole qui classe les individus. Aussi celles comme ceux qui usent de fioritures à mauvais escient, avec maladresse ou de façon ostensible ou abusive, sont-elles la cible de discours critiques. Le costume devient l'indice d'un mauvais comportement et souvent d'une vie dépravée. Il dénature. Il est le signe d'une maladie sociale et politique.

Dans des lois de contrôle social, pour les femmes et les hommes, des parures jugées outrées et portées en public caractérisent les adultères ou encore les hétaïres et les mignons. La cité, en définissant des grilles de lecture vestimentaires précises, réprouve des individus pour leur comportement. Par exemple, à Locres au VII siècle avant

42 Hésiode, Théogonie, 585.

43 Ainsi chez Pindare, Néméennes, I, 38 et 50, Héra, l'épouse légitime, est chrusothronos ("vêtue de son manteau aux fleurs d'or") tandis qu'Alcmène est apeplos ("sans peplos »). Voir Scheid \& Svenbro 2003 [1994] : 62.

44 Cf. Dalby 2002. 
notre ère, Zaleucos impose aux épouses de citoyens des déplacements contrôlés et un vêtement spécifique pour être reconnues comme telles. Les femmes qui portent bijoux et tenues bigarrées (brodées et de couleur pourpre), très travaillées, deviennent des séductrices aux mauvaises mœurs. Leur costume signale une attitude dénuée de dignité et de tempérance. Le constat est comparable pour des hommes. Au regard de tous, s'ils portent de l'or et des habits somptueux et délicats, ils ne peuvent être d'honorables citoyens :

Tous les autres n'avaient infligé que des amendes aux femmes coupables, mais lui contint leurs débordements (akolosiar) par un châtiment plein d'astuce. Voici ses prescriptions. Une femme libre ne devait pas être accompagnée par plus d'une servante à moins qu'elle ne fût ivre, ni sortir de la ville la nuit, à moins qu'elle ne fût adultère, ni se parer de bijoux d'or (mêde peritithesthai chrusia) ou d'un vêtement bordé de pourpre (mêde esthêta paruphasmenên), à moins qu'elle ne fût une hétaïre. Un homme ne devait pas porter de bague en or (daktulios hupochrusos) ni de manteau à la milésienne (bimation isomilêsion), s'il n'était hetairos ou adultère. Les exclusions infamantes qu'impliquaient ces peines lui permirent de détourner aisément du luxe nuisible et des conduites déréglées, car personne ne voulait en avouant un dérèglement infamant être la risée de ses concitoyens ${ }^{45}$.

Une mesure comparable, attribuée parfois à Dioclès ${ }^{46}$, aurait été en vigueur à Syracuse, probablement à la toute fin du Ve siècle avant notre ère $e^{47}$.

Le port de certains vêtements et ornements devient une marque d'atimie (déshonneur). Ceux-ci soulignent une infamie sociale et politique. En définissant un «costume de honte», composé d'étoffes richement ornées et colorées de pourpre, de bijoux et d'or - des «objets d'art»-, ces lois somptuaires visent à garantir l'identité des personnes relative à leur apparence, une «identité de genre », comme le remarque Pauline Schmitt Pantel ${ }^{48}$, mais aussi une identité de mœurs, sociale et politique. Inversement, dans un contexte rituel où une parure luxueuse signale le statut honorable des femmes, c'est

45 Diodore de Sicile, XII, 21, 1-2 (traduction M. Casevitz, CUF, légèrement modifiée).

46 Diodore de Sicile, XIII, 33, 2.

47 Cf. Athénée, XII, 521B.

48 Schmitt Pantel 2009 [1981] : 95-98. 
l'absence de ces ornements qui caractérise les femmes licencieuses. Ainsi, on attribue à Solon, archonte en 594 avant notre ère à Athènes, la décision d'avoir interdit aux femmes adultères de revêtir des ornements et de prendre part aux célébrations de la cité. Si elles n’obéissaient pas, elles étaient dépouillées de leurs belles parures :

[Il] interdit toute parure (ouk kosmeisthai) à la femme qui a été surprise en adultère, il lui défend de s'associer aux cérémonies sacrées publiques, de peur qu'en ne se mêlant aux femmes honnêtes elle ne les corrompe. Si, en dépit de cette défense, elle prend part à ces cérémonies, ou revêt des parures (kosmêtai), il ordonne au premier qui la rencontrera de déchirer ses vêtements (katarregnunai ta himatia), de lui arracher ses ornements (ton kosmon aphairesthai) et de lui donner des coups en évitant toutefois de la faire mourir ou de l'estropier. Le législateur frappe ainsi cette femme d'une peine déshonorante et lui prépare une vie intolérable ${ }^{49}$.

Ne pas s'habiller de belles parures, notamment dans les fêtes de la cité, est une marque infamante, un signe d'atimie. Cette mesure est politique puisqu'elle vise celles qui compromettent par leur comportement la reproduction du corps civique ${ }^{50}$.

Ces jeux de vêtements, leur instrumentalisation pour dire le bon ordre ou, au contraire, la démesure, la violence et le chaos dans la cité, sont traduits en langage visuel sur une boîte à bijoux ou à fards, une pyxide attique à figures rouges, attribuée au peintre de Meidias, datant de la fin du Ve siècle avant notre ère. Cet objet, destiné à une femme, représente des figures féminines vêtues de longues tuniques, parées de couronnes et de mitres, et portant des bijoux, s'affairant à leur mise : l'une lace sa sandale, les autres tiennent ou reçoivent un collier de perles. Il ne s'agit pas d'une banale scène de toilette. La scène peinte propose au contraire une image plus complexe et fine des parures et costumes féminins. Ainsi, Aphrodite, derrière un char, est vêtue d'un long chiton resserré à la taille par une ceinture, ses cheveux sont relevés et noués, attachés par un bandeau; elle porte des bracelets, des boucles d'oreilles et un collier de perles. Devant, comme attelage, sont figurés deux Érotes - Pothos (Désir) et $\hat{E}$ Eulogos (Au doux langage) - quasi nus : ils portent des sandales; des couronnes encadrent leur longue chevelure. Les autres

49 Eschine, I, Contre Timarque, 183.

50 Schmitt Pantel 2009 [1981]. 
personnages féminins présents sur la boîte ressemblent à la déesse. À droite de l'image, Hygieia, personnification de la santé, cueille un fruit sur un arbuste (Cf. Cahier des illustrations, figure 2). Sur la suite de la composition, Kalê (Beauté), un collier de perles entre ses mains, se trouve derrière Aphrodite; son visage est tourné vers Harmonia assise qui tient une couronne de laurier, comme les autres leur collier (Cf. Cabier des illustrations, figure 3) - Harmonia (Accord) étant ici le pendant d'Eunomia (Bon ordre) assise et placée de l'autre côté de l'image (Cf. Cabier des illustrations, figure 4). Enfin, un autre Éros, Himeros (Puissant désir), un fruit dans sa main gauche, présente de sa main droite une chaine de perles à Eudaimonia (Bonheur) qui est en train de lacer une sandale (Cf. Cabier des illustrations, figures 3 et 4). Au centre, Paidia (Jeu d'enfant) tend un collier de la main droite et tient une boite de la gauche, sur laquelle se trouve un collier ou des fruits (Cf. Cabier des illustrations, figure 4). À l'intérieur de l'image, la beauté féminine se construit dans un jeu entre ornementation du corps et objets féminins, où Éros est un acteur privilégié qui fait naitre et rayonner la beauté et circuler la séduction et le désirir ${ }^{51}$

L'ensemble rappelle les éléments qui définissent l'enkosmia : la beauté, l'harmonie, le bonheur, le jeu, le bon ordre, la santé, le désir, la séduction et des paroles agréables. En revanche, sur le couvercle de la même pyxide, on voit la mise à mort de Penthée démembré par les filles de Cadmos. Le dieu y est figuré assis et entouré de ces femmes. Leur costume n'est pas différent de celui des femmes représentées sur le corps de la boîte, à l'exception du thyrse que l'une d'elles a comme attribut. Cependant, elles s'affairent, pour les unes, à jouer de la lyre et du tambour, tandis que les autres, en mouvement, frénétiquement, courent, dansent au rythme de la musique, et portent un animal sauvage et un enfant, peut-être Penthée, plutôt que des bijoux (figure 1). Dans le mythe, en effet, Agavé, Ino et Autonoé, rendues folles par Dionysos qu'elles refusent d'accepter comme dieu, quittent leur métier à tisser pour le Cithéron, suivies par les autres femmes de la cité. Penthée, travesti, est démasqué, puis écartelé par ces femmes furieuses, parmi lesquelles se trouve sa mère, qui ne reconnait plus son propre fils. Ainsi, le décor de la pyxide dit, dans le domaine d'Aphrodite, la félicité

51 Voir Lissarrague 2011. 
attendue, et s'oppose au déchaînement de violence féminin, au diasparagmos (mise en pièce), dans la sphère de Dionysos, à la folie et au désordre, figurés sur le couvercle de la même boîte.

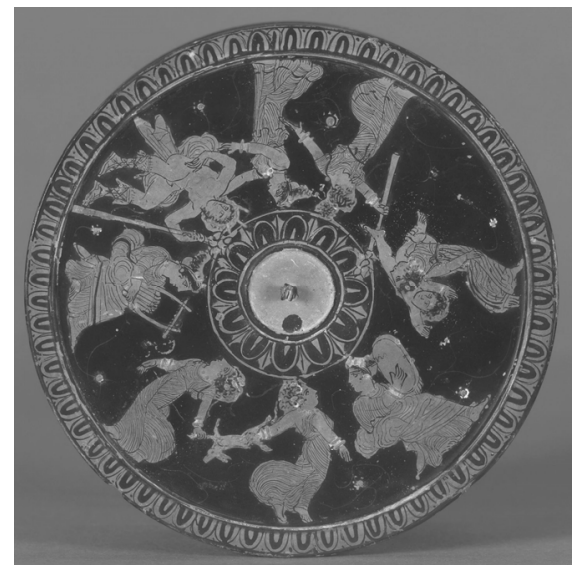

Figure 1. Dionysos entouré de ménades. Pyxide attique à figures rouges attribuée au peintre de Meidias, vers 420-400 avant notre ère. Terre cuite, Londres, British Museum, E775 (C) The Trustees of the British Museum.

Le kosmos donne une belle apparence et, parfois, plus de valeur. Il a dès lors aussi une signification politique et religieuse. La variété des parures, leur brillance, leurs couleurs (notamment l'alliance de la pourpre et de l'or, les deux couleurs les plus prestigieuses), les bigarrures et les fleurs disent précisément ce qui est beau, l'eukosmia. Néanmoins, l'agencement ordonné ne produit l'effet d'éclat escompté, la charis, que s'il répond aux normes attendues, sociales, politiques et religieuses, en termes d'espace et de manière d'être. Le costume, vêtements et parures, parce qu'il énonce l'ordre, l'harmonie et la beauté, caractérise un idéal de conduite sociale. Néanmoins, comme tenue désassortie et/ou décalée, il dénature, défigure, enlaidit et désigne de ce fait un mauvais comportement, l'indécence, une attitude atypique susceptible d'être condamnée, le désordre et la violence.

Analyser le costume féminin suivant une approche historique et anthropologique met en exergue que, loin d'être futile et superficiel, le vêtement est essentiel dans des sociétés où les actes sont aussi importants que le paraitre. Le costume et ses ornementations, leur 
assemblage et ordonnancement, sont autant de manières de dire, de se montrer, d'agir et de provoquer. Ainsi, il y a coïncidence entre la femme et son vêtement. Le corps paré est doté d'un pouvoir agissant et c'est bien cet effet produit qui est jaugé par les Anciens, en particulier quand ils critiquent les parures et artifices, ou encore quand ils légifèrent pour mieux canaliser la nature féminine, contrôler ces corps vêtus ou bien les utiliser au profit de la communauté pour promouvoir l'enkosmia d'une célébration (par exemple, dans des processions publiques où se distinguent des personnages parés d'or et aux vêtements fleuris ou bordés de pourpre) ou la préserver (en imposant un dénuement cultuel par une interdiction de la poikilia des tenues vestimentaires des femmes dans des cultes en l'honneur de Déméter ou Despoinia).

Les discours élaborés par les poètes, les historiens, les législateurs, etc., de l'époque archaïque à l'époque hellénistique, instrumentalisent le costume. Ils montrent que les vêtements féminins servent une mise en beauté. Ceux-ci disent la beauté des femmes et l'intensifient à condition d'être bien utilisés, avec mesure. Aussi, quand le costume fait corps avec ces dernières, en est-il un prolongement naturel. Cependant les parures féminines sont également ambivalentes. Elles sont, à la fois, beauté et promesse de félicité ou encore disent la ruse, la menace et la violence. Le costume, parce qu'il orne le corps, le travaille, le modèle, pour l'embellir ou mieux le cacher, définit ainsi la femme entre pudeur, séduction érotique, beauté et intelligence rusée, entre norme et transgression. En effet, il ne s'agit pas seulement d'une beauté physique et vestimentaire. Le costume doit être conforme à un comportement attendu, fonction de l'activité, du statut, du sexe, de l'âge, des lieux et des occasions privées et publiques. Dans cette perspective, le costume - que l'on revêt par choix ou conformément à la loi et/ou sous la contrainte - participe à un mode efficace de construction de la pensée et du monde pour dire l'excellence et l'harmonie ou au contraire le désordre social et politique dans les cités grecques. 


\section{Sources}

Anthologie palatine, VII, Paris, Les Belles Lettres, «CUF », $2002^{2}$.

Aristophane, Assemblée des femmes, Paris, Les Belles Lettres, «CUF », 19772.

Aristophane, Les Thesmophories, Paris, Les Belles Lettres, «CUF », $1942^{2}$.

Aristophane, Lysistrata, Paris, Les Belles Lettres, «CUF», $1940^{2}$.

Aristophane, Ploutos, Paris, Les Belles Lettres, «CUF», 19772.

ATHÉNÉE, Les Deipnosophistes, XII (The Learned Banqueters, Books 12-12, 594b), LondresCambridge, «LCL », 2010.

CléArchos apud AtHÉnÉe, Les Deipnosophistes, XIII (The Learned Banqueters, Books 13.594b-14), Londres-Cambridge, «LCL », 2010.

Diodore De Sicile, La Bibliothèque historique, XII, Paris, Les Belles Lettres, La roue à livres, $2002^{2}$.

Diodore DE SICILE, La Bibliothèque historique, XIII, (Library of History, V [XII, 41-XIII]), Londres-Cambridge, «LCL », 1950.

Diogène LAËrCE, Vies et doctrines des philosophes illustres, Paris, « La pochothèque », Le livre de poche, 1999.

Douris de SAmos apud ATHÉNÉE, XII (The Learned Banqueters, Books 12-12, 594b), Londres, Cambridge, LCL, 2010.

ÉLIEN, Histoires variées, Paris, Les Belles Lettres, La roue à livres, 1991.

ÉPHORE apud Diogène LAËrCE, Vie, doctrines et sentences des philosophes illustres, Paris, «La pochothèque », Le livre de poche, 1999.

EsChine, I, Contre Timarque, Paris, Les Belles Lettres, «CUF», 1927.

EURIPIDE, Andromaque, Paris, Les Belles Lettres, «CUF », 19972.

EuripIDE, Les Bacchantes, Paris, Les Belles Lettres, «CUF», $1998^{2}$.

Fragments et lettres de Theano, de Périctioné, Pbintys, Mélissa et Mya, Paris, L'artisan du livre, 1932.

Hérodote, Histoires, Paris, Les Belles Lettres, «CUF», 1992.

HÉsIode, Les Travaux et les jours, Paris, Les Belles Lettres, « CUF », 1928.

HÉsIODE, Théogonie, Paris, Les Belles Lettres, «CUF », 1928.

HOMÈRE, Iliade, XIV, Paris, Les Belles Lettres, « CUF », 1938.

Hymne homérique à Aphrodite I, Paris, Les Belles Lettres, «CUF», 19976.

Hymne homérique à Aphrodite II, Paris, Les Belles Lettres, «CUF», 19976.

JAmBLIQUE, Vie de Pythagore, Paris, Les Belles Lettres, La roue à livres, 2011. 
Myron de PriÈne apud AtHénÉe, Les Deipnosophistes, XIV (The Learned Banqueters, Books 13.594b-14), Londres, Cambridge, «LCL », 2010.

Pindare, Les Néméennes, I, Paris, Les Belles Lettres, «CUF », 19674.

PlutARQue, Lycurgue, Paris, Les Belles Lettres, «CUF », 19933.

Xénophon, Économique, Paris, Les Belles Lettres, «CUF », 1949.

Xénophon, Mémorables, Paris, Les Belles Lettres, «CUF », 2011.

[XÉNOPHON], Constitution des Athéniens, Paris, Les Belles Lettres, La roue à livres, 2008.

Règlement cultuel d'Érésos, ve siècle avant notre ère: LSCGS, n³2 (LSCGS: SOKOLOWSKI Franciszek, Lois sacrées des cités grecques. Supplément, Paris, De Boccard, 1962).

Règlement cultuel de Lycosoura, III ${ }^{\text {e }}$ siècle avant notre ère : IG V 2, 514 (IG: Inscriptiones Graecae, publiées par l'Académie de Berlin, 1903-); LSCG, nº8 (LSCG: SOKOLOWSKI Franciszek, Lois sacrées des cités grecques, Paris, De Boccard, 1969).

Règlement cultuel de Patras, III $^{\text {e }}$ siècle avant notre ère: LSCGS, n³3 A (LSCGS : SOKOLOWSKI Franciszek, Lois sacrées des cités grecques. Supplément, Paris, De Boccard, 1962).

Règlement cultuel des mystères d'Andania, Ir siècle avant notre ère : IG IV, 1, 1390 (IG : Inscriptiones Graecae, publiées par l'Académie de Berlin, 1903-).

\section{Bibliographie}

BARKer Albert W., 1922, «Domestic Costume of the Athenian Woman in the Fifth and the Fourth Centuries B.C. ", American Journal of Archaeology, 26, p. 410-425.

BARTHES Roland, 1957, "Histoire et sociologie du vêtement», Annales ESC, 3, p. $430-441$.

BeCtARTE Hélène, 2009, «Le costume de l'épouse dans l'art funéraire attique de l'époque classique», in Lydie Bodiou, Véronique MeHL, Jacques OulHeN, Francis Prost \& Jérôme WiLgaux (dir.), Chemin faisant. Mythes, cultes et société en Grèce ancienne, Rennes, Presses universitaires de Rennes, p. 236-238.

Bruit Zaidman Louise, 1996, «Le temps des jeunes filles dans la cité grecque: Nausicaa, Phrasikleia, Timareta et les autres ", Clio. Histoire, Femmes et Sociétés, 4, Le temps des jeunes filles, p. 33-50.

BrulÉ Pierre, 1987, La fille d'Athènes. La religion des filles à Athènes à l'époque classique. Mythes, cultes et societé, Paris, Les Belles Lettres.

CASEVITZ Michel, 1989, «À la recherche du kosmos», Le temps de la réflexion, X, p. 97-119. 
Cartledge Paul, Millet Paul \& Sitta von Reden (eds), 1998, Kosmos. Essays in Order, Conflict and Community in Classical Athens, Cambridge, CUP.

Cleland Liza, Harlow Mary \& Lloyd Lewellyn-Jones (eds), 2005, The Clothed Body in Ancient World, Oxford, Oxbow Books.

Cleland Liza, 2009, "A Hierarchy of Women: Status, Dress and Social Construction at Andania », http://edinburgh.academia.edu/LizaCleland

DAlby Andrew, 2002, «Levels of Concealment: the Dress of Hetairai and Pornai in Greek Texts ", in Lloyd LLEWELlyn-Jones (ed.), Women's Dress in the Ancient Greek World, Londres, Duckworth, p. 111-124.

Deshours Nadine, 2006, Les mystères d'Andania. Étude d'épigraphie et d'bistoire religieuse, Paris-Bordeaux, Ausonius.

Dillon Matthew, 1997, Pilgrims and Pilgrimage in Ancient Greece, Londres, Routledge.

—, 2002, Girl and Women in Classical Greek Religion, Londres, Routledge.

DuCAT Jean, 1974, «Le mépris des hilotes », Annales ESC, 29, p. 1451-1464.

GHERCHANOC Florence, 2003, «Les atours féminins des hommes: quelques représentations du masculin/féminin dans le monde grec antique. Entre initiation, ruse, séduction et grotesque, surpuissance et déchéance », Revue bistorique, $\mathrm{CCCV} / 4$, p. 739-791.

—, 2008, "Nudités athlétiques et identités en Grèce ancienne», in Florence GHERCHANOC \& Valérie HUET (dir.), S'babiller, se déshabiller dans les mondes anciens, Mètis, n.s. 6, p. 75-101.

—, 2011, « Maquillage et identité : du visage au masque, de la décence à l’outrage, de la parure à l'artifice ", in Lydie Bodiou, Florence GHerchanoc, Valérie HuET \& Véronique MeHL (dir.), Parures et artifices: le corps exposé dans l'Antiquité, Paris, L'Harmattan, p. 23-44.

—, 2012, L'oikos en fête. Célébrations familiales et sociabilité en Grèce ancienne, Paris, Publications de la Sorbonne.

GHERCHANOC Florence \& Valérie HUET, 2007, « S’habiller et se déshabiller en Grèce et à Rome. Pratiques politiques et culturelles du vêtement. Essai historiographique », Revue Historique, CCCIX/1, p. 3-30.

GRAND-CLÉMENT Adeline, 2011a, «Du bon usage du vêtement bariolé en Grèce ancienne », in Lydie Bodiou, Florence Gherchanoc, Valérie Huet \& Véronique MeHL (dir.), Parures et artifices: le corps exposé dans l'Antiquité, Paris, L'Harmattan, p. 255-274.

—, 2011b, La fabrique des couleurs. Histoire du paysage sensible des Grecs (VIIIe-début du Ve s. av. n.è.), Paris, De Boccard. 
IRIARTE Ana, 2006, «Le genre des habits et le tissage de la nudité en Grèce ancienne », in Violaine Sebillotte Cuchet \& Nathalie ERnoult (dir.), Problèmes du genre en Grèce ancienne, Paris, Publications de la Sorbonne, p. 289-301.

JEAMMET Violaine, 2003, «Le costume grec à travers les figurines en terre cuite: reflet d'une société démocratique ? ", in François CHAUSSON \& Hervé INGLEBERT (dir.), Costume et société dans l'Antiquité et au haut Moyen-Agge, Paris, Picard, p. 25-36.

Jones Christopher, 1999, «Processional Colors », in Bettina Bergmann \& Christina KondoleOn (eds), "The Art of Ancient Spectacle », Studies in the History of Art, 56 , p. 247-257.

JosT Madeleine, 1985, Sanctuaires et cultes d'Arcadie, Paris, De Boccard.

LE GUEN-POLLET Brigitte, 1991, La vie religieuse dans le monde grec du Ve au III siècle avant notre ère, Toulouse, Presses universitaires du Mirail.

Lissarrague François, 2011, «Éros en tête : femme, miroir et bijoux en Grèce ancienne", in Lydie Bodiou, Florence GHerchanoc, Valérie HuET \& Véronique MeHL (dir.), Parures et artifices: le corps exposé dans l'Antiquité, Paris, L'Harmattan, p. 15-22.

Llewellyn-Jones Lloyd (ed.), 2002, Women's Dress in the Ancient Greek World, Londres, Duckworth.

LLEWELlyn-Jones Lloyd, 2003, Aphodite's Tortoise. The Veiled Woman of Ancient Greece, Swansea, The Classical Press of Wales.

Loraux Nicole, 1989, Les expériences de Tirésias. Le féminin et l'homme grec, Paris, Gallimard.

—, 2009 [2003], « Mélissa, épouse et fille de tyran », in Nicole LorAux (dir.), La Grèce au féminin, Paris, Les Belles Lettres, p. 3-37.

LOSFELD Georges, 1991, Essai sur le costume grec, Paris, De Boccard.

MoRIzot Yvette, 2001, "Le vêtement grec dans sa matérialité : découvertes et recherches récentes ", Histoire de l'art, 48, p. 11-22.

Pirenne-Delforge Vinciane, 2001, « Prairie de Pandore et jardin d'Aphrodite. Le

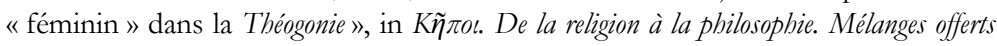
à A. Motte, Kernos, supplément 11, p. 86-99.

Roccos Linda J., 1995, «The Kanephoros and her Festival Mantle in Greek Art », American Journal of Archaeology, 99, p. 641-666.

SCHeID John \& Jesper Svenbro, 2003 [1994], Le métier de Zeus. Mythe du tissage et du tissu dans le monde gréco-romain, Paris, Errance.

Schmitt Pantel Pauline, 2009 [1981], «L'âne, l’adultère et la cité », in ead., Aitbra et Pandora. Femmes, Genre et Cité dans la Grèce antique, Paris, L'Harmattan, p. 91-98. 
Vernant Jean-Pierre, 2007 [1996], «Les semblances de Pandora », in CEuvres II, Paris, Le Seuil, p. 2032-2043.

VIDAL-NAQUET Pierre, 1983 [1970], «Valeurs religieuses et mythiques de la terre et du sacrifice dans l'Odyssée ", in id., Le chasseur noir. Formes de pensées et formes de société dans le monde grec, Paris, La Découverte/Maspéro, p. 39-68.

Villanueva Puig Marie-Christine, 1992, Images de la vie quotidienne en Grèce ancienne, Paris, Hachette.

Will Edouard, 1955, Korinthiaka. Recherches sur l'bistoire et la civilisation de Corinthe des origines aux guerres médiques, Paris, De Boccard. 